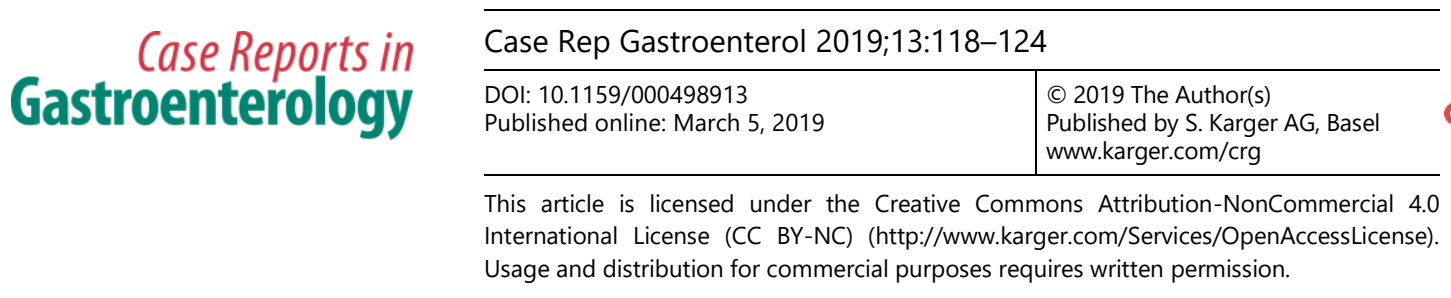

\title{
EpCAM- and/or NCAM-Expressing Hepatocellular Carcinoma in Which Behavior of Hepatic Progenitor Cell Marker-Positive Cells Are Followed
}

\author{
Atsunori Tsuchiya $^{a} \quad$ Takeshi Suda $^{b} \quad$ Chiyumi Oda $^{a} \quad$ Atsushi Kimura $^{a}$ \\ Kazunori Hosaka ${ }^{a}$ Naruhiro Kimura ${ }^{a}$ Kentaro Tominaga ${ }^{a}$ \\ Kazunao Hayashi ${ }^{a}$ Masaaki Takamura ${ }^{a}$ Shuji Terai ${ }^{a}$ \\ aDivision of Gastroenterology and Hepatology, Graduate School of Medical and Dental \\ Science, Niigata University, Niigata, Japan; ${ }^{b}$ Department of Gastroenterology and \\ Hepatology, Uonuma Institute of Community Medicine, Niigata Medical and Dental \\ Hospital, Niigata, Japan
}

\author{
Keywords \\ EpCAM · NCAM · Hepatocellular carcinoma · Metastasis · Vascular invasion
}

\begin{abstract}
Hepatic progenitor cell (HPC) marker-positive hepatocellular carcinomas (HCCs) have recently been extensively analyzed, and their prognosis has been reported as poor compared to HPC marker-negative HCCs. However, previous studies have analyzed the existence of HPC markerpositive cancer cells only in primary lesions, as well as the recurrence rate and prognosis of such tumors. Here, we are the first to report the behavior of HPC marker-positive cancer cells during vascular invasion and metastasis of an HCC. We concurrently analyzed EpCAM- and/or NCAM-expressing cancer cells in the primary, vascular invasion, and metastatic lesions of an HCC. An HCC which includes EpCAM- and/or NCAM-expressing cancer cells has not been previously reported. EpCAM- and/or NCAM-positive cancer cells invaded the vessels and formed heterogeneous populations of these HPC marker-positive cancer cells with HPC marker-negative cancer cells. The frequency of HPC marker-positive cancer colonies and cells in vessels was higher than that in the primary HCC. In the metastatic lesions, EpCAM-positive cancer cells
\end{abstract}




\section{Case Reports in Gastroenterology}

Case Rep Gastroenterol 2019;13:118-124

DOI: $10.1159 / 000498913$

(c) 2019 The Author(s). Published by S. Karger AG, Basel www.karger.com/crg

Tsuchiya et al.: EpCAM- and/or NCAM-Expressing Hepatocellular Carcinoma in Which Behavior of Hepatic Progenitor Cell Marker-Positive Cells Are Followed

were more frequently detected than NCAM-positive cancer cells, indicating that EpCAM may be more important than NCAM for cancer cell settlement in the metastatic lesions. Furthermore, bigger metastatic tumors tended to include HPC marker-positive cancer cells, suggesting that HPC marker-positive cancer cells have a growth advantage in the metastatic lesions. These results showed that HPC marker-positive cancer cells would be important for vascular invasion and metastasis and suggested that HPC marker-positive cancer cells are an important target in $\mathrm{HCC}$ treatment.

(C) 2019 The Author(s)

Published by S. Karger AG, Basel

\section{Introduction}

Hepatic progenitor cells (HPCs), which are involved in ductular reactions during chronic liver injury or severe acute liver damage [1], are thought to have a crucial role in the production and supply of new hepatocytes [2]. During liver regeneration from HPCs to hepatocytes, cells such as hematopoietic cells or myofibroblasts that surround HPCs, as well as the extra cellular matrix, affect the regeneration process including matrix degradation [3], cell fate decisions [4], and cell expansion [5], migration [6] and differentiation [7]. During these steps, HPCs express many HPC-specific markers such as epithelial cell adhesion molecule (EpCAM) [8] and neural cell adhesion molecule (NCAM) [9]. These markers are transiently upregulated only during the specific period of regeneration and rapidly disappear during the differentiation steps. Recently, these HPC markers have received attention due to their appearance in liver cancer, especially in hepatocellular carcinoma (HCC). These HPC marker-positive cancer cells are detected in various proportions and comprise a heterogeneous population of cancer cells. These HPC marker-positive HCCs have been reported to be more highly associated with characteristics of poor prognosis such as early metastasis and rapid expansion compared to the conventional HPC marker-negative HCCs [10]. While there is still much debate as to whether these HPC marker-positive cancer cells are truly cancer stem cells or not, at least these HPC-positive cancer cells would exert the function associated with each expressed HPC marker. However, whether HPC marker-positive cancer cells really do enhance metastasis and expansion, and how these HPC marker-positive cancer cells distribute to the metastatic lesions have not yet been clinically elucidated. In this case report, we report the first case of an EpCAM- and/or NCAM-expressing HCC. Although each individual marker has been reported to relate to poor prognosis, both markers expressing HCC have not yet been reported. Furthermore, we were able to analyze the primary lesions, the vascular invaded lesions and the metastatic lesions at the same time and could check the frequency at which the HPC markerpositive cancer cells were included in blood vessels and in metastatic lesions. This is the first reported case to show the heterogeneous distribution of HPC marker-positive cancer cells, and their greater importance for tumor metastasis and expansion in human.

\section{Case Report}

A 65-year-old man was transferred to our hospital due to treatment of a huge HCC that occupied most of the right lobe of the liver. He was an asymptomatic hepatitis B virus carrier and had a history of prostate enlargement, atrial fibrillation, and diabetes mellitus. Physical examination revealed hepatomegaly. Laboratory data revealed slight elevation of transaminase and biliary enzymes probably due to the large size of the tumor. While the level of $\alpha$ fetoprotein (AFP) was normal, the level of des- $\gamma$-carboxy prothrombin was 10,225 mAU/mL 


\section{Case Reports in Gastroenterology}

Case Rep Gastroenterol 2019;13:118-124

DOI: 10.1159/000498913

(c) 2019 The Author(s). Published by S. Karger AG, Basel www.karger.com/crg

Tsuchiya et al.: EpCAM- and/or NCAM-Expressing Hepatocellular Carcinoma in Which Behavior of Hepatic Progenitor Cell Marker-Positive Cells Are Followed

(normal values, $<40 \mathrm{mAU} / \mathrm{mL}$ ). Contrast-enhanced computed tomography was performed, revealing a huge HCC that occupied most of the right lobe of the liver, as well as some very small metastatic nodules that were suspected in the left lobe of the liver (Fig. 1a). Surgical resection of the huge tumor was chosen as the treatment, due to difficulties with controlling such a large tumor and in order to avoid HCC rupture. The surgery proceeded after informed consent. During the operation, surgeons first resected the right lobe HCC, and then as many of the small nodules at the surface of the left lobe of the liver as possible. Pathological examination revealed that the tumor was a moderately to poorly differentiated HCC with portal vein invasion. Tissues of the primary lesion were immunohistochemically analyzed for NCAM and EpCAM separately, which revealed that $10-20 \%$ and $5-10 \%$ cells were positive for NCAM and EpCAM, respectively. Protein expression was defined using the following scale, based on the percentage of tumor cells expressing the specific protein: -, 0\%;, $\pm 0-5 \% ; 1+, 5-10 \% ; 2+, 10-20 \% ; 3+$, $20-50 \% ; 4+,>50 \%$. By using this scale, the tumor in the primary lesion was characterized as NCAM2+ and EpCAM1+ (Fig. 1b). In this HCC case, we were also able to immunostain the vascular-invaded lesions and the metastatic lesions using the same antibodies separately and to analyze how frequently these HPC marker-positive cancer cells were included in the vessels or in the metastatic lesions. We checked the following two points regarding the vascular invaded lesions: (1) the ratio of the number of vessels invaded by specific HPC marker-positive cancer cells/the total number of vessels invaded by cancer cells (expressed as \%), and (2) the ratio of specific HPC marker-positive cancer cells in the vessels/the number of total cancer cells in the vessels (expressed as \%). Regarding 1, the number of vessels invaded by specific HPC marker-positive cancer cells/the total number of vessels invaded by cancer cells was $5 / 7$ (71.4\%) for NCAM- and 6/9 (66.7\%) for EpCAM-positive cancer cells. Regarding 2: the number of specific HPC marker-positive cancer cells in the vessels/the number of total cancer cells in the vessels was 34/134 (24.3\%) for NCAM- and 42/140 (30.0\%) for EpCAM-positive cells (Fig. 1b and 2). These results revealed that the HPC marker-positive cancer cells had invaded the vessels together with HPC marker-negative cancer cells forming a heterogeneous population of cancer cells. Additionally, EpCAM- and/or NCAM-expressing cancer cell populations in particular had invaded the vessels of this tumor at a high rate. Finally, we analyzed the eight metastatic tumors with regard to the size of the tumor and the frequency of each marker according to the above definitions. The results for the four biggest tumors were as follows (in order, starting from the biggest tumor): (1) $17 \times 10 \mathrm{~mm}$, NCAM2+, EpCAM2+; (2) $8 \times 4 \mathrm{~mm}$, NCAM \pm , EpCAM4+; (3) $7 \times 6$ mm, NCAM \pm , EpCAM4+; (4) $4 \times 2$ mm, NCAM-, EpCAM4+. The other four smaller tumors, which were of the following size: (5) $2 \times 2 \mathrm{~mm}$, (6) $2 \times 2 \mathrm{~mm}$, (7) 2 $\times 2 \mathrm{~mm}$, and (8) $2 \times 1 \mathrm{~mm}$, did not express any markers (Fig. 2). These results clearly indicated that the bigger tumors were HPC marker-positive and that a greater number of EpCAM-positive than NCAM-positive cancer cells were present in the metastatic lesions, whereas a greater number of NCAM-positive than EpCAM-positive cancer cells were present in the primary tumor.

\section{Discussion}

In this case report, by concurrent analysis of EpCAM- and/or NCAM-expressing HCCs in the primary lesion, in vascular invaded lesions, and in metastatic lesions of an HCC, we have described for the first time the behavior of EpCAM- and/or NCAM-expressing HCCs and have shown that such HPC marker-positive cancer cells were important for vascular invasion and metastasis [11]. Our group has previously reported that by using 251 operated HCC tissues 


\section{Case Reports in Gastroenterology}

Case Rep Gastroenterol 2019;13:118-124

DOI: 10.1159/000498913

(c) 2019 The Author(s). Published by S. Karger AG, Basel www.karger.com/crg

Tsuchiya et al.: EpCAM- and/or NCAM-Expressing Hepatocellular Carcinoma in Which Behavior of Hepatic Progenitor Cell Marker-Positive Cells Are Followed

and immunostaining four HPC markers EpCAM, NCAM, CK19, and DLK1, 18.3, 7.1, 14.3, and 8.0\% patients were found to have high levels of DLK1, NCAM, EpCAM, and CK19 in tumors, respectively, and the expression of two or more HPC markers was a significant predictor of poor HCC outcome, and serum levels of AFP/AFP-L3 correlated with the expression of HPC proteins. However, HPC marker-targeted therapy is not established yet. Furthermore, we found that EpCAM expression, des- $\gamma$-carboxy prothrombin $\geq 300 \mathrm{mAU} / \mathrm{mL}$, age $\geq 60$ years, and Child-Pugh class B or C were independent prognostic factors of poor outcome and were used in a new scoring system for HCC prognosis after operation [12,13]. Matthai and Ramakrishna [14] reported the frequency of EpCAM-expressing HCC. Thirty-three (41.8\%) of the 79 HCCs, comprising $16(32.7 \%)$ tumors in the surgical group and 17 (56.7\%) in the autopsy group, showed positive staining for EpCAM. By analysis of the vascular cancer-invaded lesions, we found that approximately $70 \%$ of the cancer-invaded vessels included EpCAM- and/or NCAMpositive cancer cells and that EpCAM- or NCAM-positive cancer cells represented 25-30\% of the total number of cancer cells that had invaded vessels. Given that the percentage of cancer cells in the primary tumor that expressed NCAM was 10-20\% (NCAM2+), and that expressed EpCAM was 5-10\% (EpCAM1+), these results clearly showed that both marker-positive cancer cells displayed greater vascular invasion than HPC marker-negative cancer cells. We also observed an intriguing behavior of HPC marker-positive cancer cells in metastatic lesions. In the present study, by chance we were able to analyze eight metastatic lesions at the same time, which revealed that metastatic lesions are also composed of heterogeneous populations of HPC marker-positive and -negative cancer cells and that the frequency of HPC marker-positive cancer cells differs according to the marker. Although the frequency of the HPC marker-positive cancer cells differed, this case clearly showed that the bigger metastatic tumors tended to include HPC marker-positive cancer cells. Furthermore, this case also suggested that a greater number of EpCAM-positive cancer cells than NCAM-positive cancer cells were found in metastatic lesions. This result is consistent with the recently published meta-analysis where overexpression of EpCAM was confirmed as an unfavorable predictor of the shorter overall survival and disease-free survival in HCC patients [15]. By analysis of normal HPCs, we recently reported that the addition of polysialic acid (polySia) to NCAM changes the function of NCAM from adhesion to anti-adhesion. This is because the high water binding capacity of polySia results in a high molecular volume that induces negative regulation of cell adhesion mediated by NCAM. Interestingly, this case clearly showed that, although NCAM-positive cancer cells can easily metastasize, it is however more difficult for them to settle in metastatic lesions compared to the EpCAM-positive cancer cells. PolySia on NCAM might prevent NCAM-positive cancer cells from settling in metastatic lesions. Further analysis is required to determine if the same system is operating for the NCAM-positive HCCs. We suspect that, since each markerpositive cancer cell has a different role during metastasis and this HCC expressed both markers, the tumor functions of both HPC markers were operational and thus the tumor showed further malignant behavior. We therefore believe that a therapeutic strategy that is focused on a better knowledge of the characteristics of individual HPC marker-positive cancer cells and the development of an HPC marker-targeted therapy would improve the prognosis of HCC patients.

\section{Statement of Ethics}

Informed consent was obtained from the patient for the publication of this report. This study has been approved by the institute's committee on human research. 


\section{Case Reports in Gastroenterology}

\begin{tabular}{l|l}
\hline DOI: 10.1159/000498913 & @ 2019 The Author(s). Published by S. Karger AG, Basel \\
\hline
\end{tabular}
www.karger.com/crg

Tsuchiya et al: EpCAM- and/or NCAM-Expressing Hepatocellular Carcinoma in Which Behavior of Hepatic Progenitor Cell Marker-Positive Cells Are Followed

\section{Disclosure Statement}

The authors declare no conflict of interest.

\section{Funding Sources}

There was no grant support for this paper.

\section{References}

1 Roskams TA, Theise ND, Balabaud C, Bhagat G, Bhathal PS, Bioulac-Sage P, et al. Nomenclature of the finer branches of the biliary tree: canals, ductules, and ductular reactions in human livers. Hepatology. 2004 Jun;39(6):1739-45.

2 Espanol-Suner R, Carpentier N, Van Hul V, Legry Y, Achouri S, Cordi P, et al. Liver progenitor cells yield functional hepatocytes in response to chronic liver injury in mice. Gastroenterology. 2012 Dec;143(6):15641575 e7.

3 Sakaida I, Terai S, Yamamoto N, Aoyama K, Ishikawa T, Nishina H, et al. Transplantation of bone marrow cells reduces CCl4-induced liver fibrosis in mice. Hepatology. 2004 Dec;40(6):1304-11.

4 Boulter L, Govaere 0, Bird TG, Radulescu S, Ramachandran P, Pellicoro A, et al. Macrophage-derived Wnt opposes Notch signaling to specify hepatic progenitor cell fate in chronic liver disease. Nat Med. 2012 Mar;18(4):572-9.

5 Bird TG, Lu WY, Boulter L, Gordon-Keylock S, Ridgway RA, Williams MJ, et al. Bone marrow injection stimulates hepatic ductular reactions in the absence of injury via macrophage-mediated TWEAK signaling. Proc Natl Acad Sci USA. 2013 Apr;110(16):6542-7.

6 Tsuchiya A, Lu WY, Weinhold B, Boulter L, Stutchfield BM, Williams MJ, et al. Polysialic acid/neural cell adhesion molecule modulates the formation of ductular reactions in liver injury. Hepatology. 2014 Nov;60(5):1727-40.

7 Kamiya A, Kinoshita T, Ito Y, Matsui T, Morikawa Y, Senba E, et al. Fetal liver development requires a paracrine action of oncostatin M through the gp130 signal transducer. EMBO J. 1999 Apr;18(8):2127-36.

8 Okabe M, Tsukahara Y, Tanaka M, Suzuki K, Saito S, Kamiya Y, et al. Potential hepatic stem cells reside in EpCAM+ cells of normal and injured mouse liver. Development. 2009 Jun;136(11):1951-60.

9 Tsuchiya A, Kamimura H, Takamura M, Yamagiwa S, Matsuda Y, Sato Y, et al. Clinicopathological analysis of CD133 and NCAM human hepatic stem/progenitor cells in damaged livers and hepatocellular carcinomas. Hepatol Res. 2009 Nov;39(11):1080-90.

10 Durnez A, Verslype C, Nevens F, Fevery J, Aerts R, Pirenne J, et al. The clinicopathological and prognostic relevance of cytokeratin 7 and 19 expression in hepatocellular carcinoma. A possible progenitor cell origin. Histopathology. 2006 Aug;49(2):138-51.

11 Tsuchiya A, Kamimura H, Tamura Y, Takamura M, Yamagiwa S, Suda T, et al. Hepatocellular carcinoma with progenitor cell features distinguishable by the hepatic stem/progenitor cell marker NCAM. Cancer Lett. 2011 Oct;309(1):95-103.

12 Terris B, Cavard C, Perret C. EpCAM, a new marker for cancer stem cells in hepatocellular carcinoma. J Hepatol. 2010 Feb;52(2):280-1.

13 Seino S, Tsuchiya A, Watanabe Y, Kawata Y, Kojima Y, Ikarashi S, et al. Clinical outcome of hepatocellular carcinoma can be predicted by the expression of hepatic progenitor cell markers and serum tumour markers. Oncotarget. 2018 Apr;9(31):21844-60.

14 Matthai SM, Ramakrishna B. Cancer stem cells in hepatocellular carcinoma-an immunohistochemical study with histopathological association. Indian J Med Res. 2015 Oct;142(4):391-8.

15 Zhou L, Zhu Y. The EpCAM overexpression is associated with clinicopathological significance and prognosis in hepatocellular carcinoma patients: A systematic review and meta-analysis. Int J Surg. 2018 Aug;56:27480 . 


\section{Case Reports in Gastroenterology}

\begin{tabular}{l|l}
\hline Case Rep Gastroenterol 2019:13:118-124 \\
\hline DOI: 10.1159/000498913 & $\begin{array}{l}\text { (c) 2019 The Author(s). Published by S. Karger AG, Basel } \\
\text { www.karger.com/crg }\end{array}$ \\
\hline
\end{tabular}

Tsuchiya et al: EpCAM- and/or NCAM-Expressing Hepatocellular Carcinoma in Which Behavior of Hepatic Progenitor Cell Marker-Positive Cells Are Followed

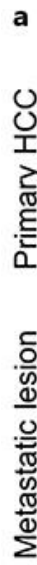

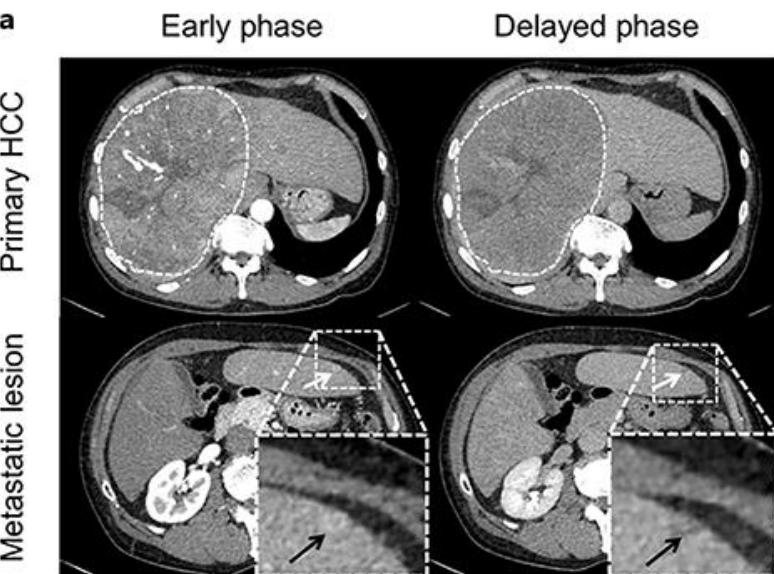

EpCAM

NCAM
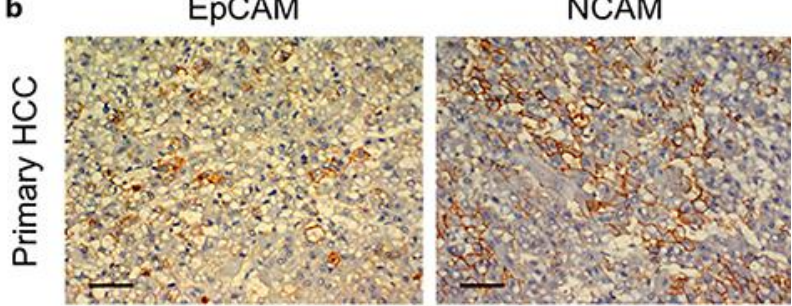

$\frac{\sqrt{2}}{\frac{2}{2}}$
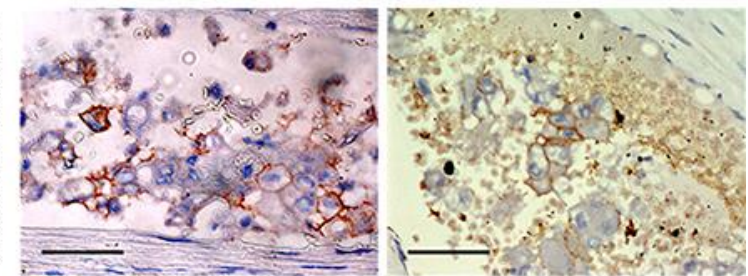

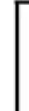
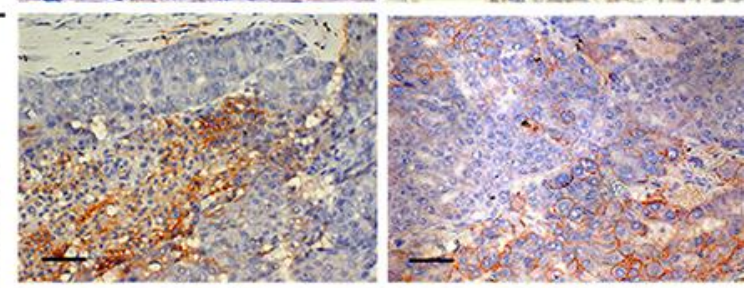

오

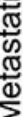
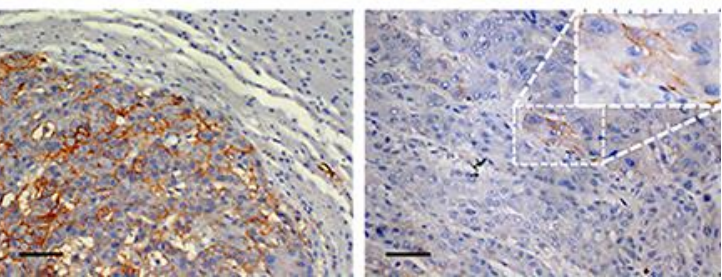
Fig. 1. Hepatocellular carcinoma (HCC) before operation. a Contrast-enhanced computed tomography of the HCC. A huge HCC occupied most of the right lobe of the liver (dashed white lines circle the HCC). Small metastatic lesions (white and black arrows) were suspected at the surface of the left lobe of the liver. $\mathbf{b}$ Histochemical analysis of EpCAM and NCAM expression in the primary and metastatic tumor lesions and in the blood vessels. The tissues were counterstained with hematoxylin. In the primary lesions, 5-10\% of cancer cells were EpCAM positive and 10-20\% of cancer cells were NCAM positive (uppermost panels). In the blood vessels, cancer cells that were heterogeneously stained for EpCAM and NCAM were frequently detected (panels second from the top). In the metastatic lesions (bottom four panels), heterogeneously stained HPC marker-positive tumors were also detected; however, the frequency of HPC-positive cancer cells differed according to the tumor. Scale bar $=50 \mu \mathrm{m}$.

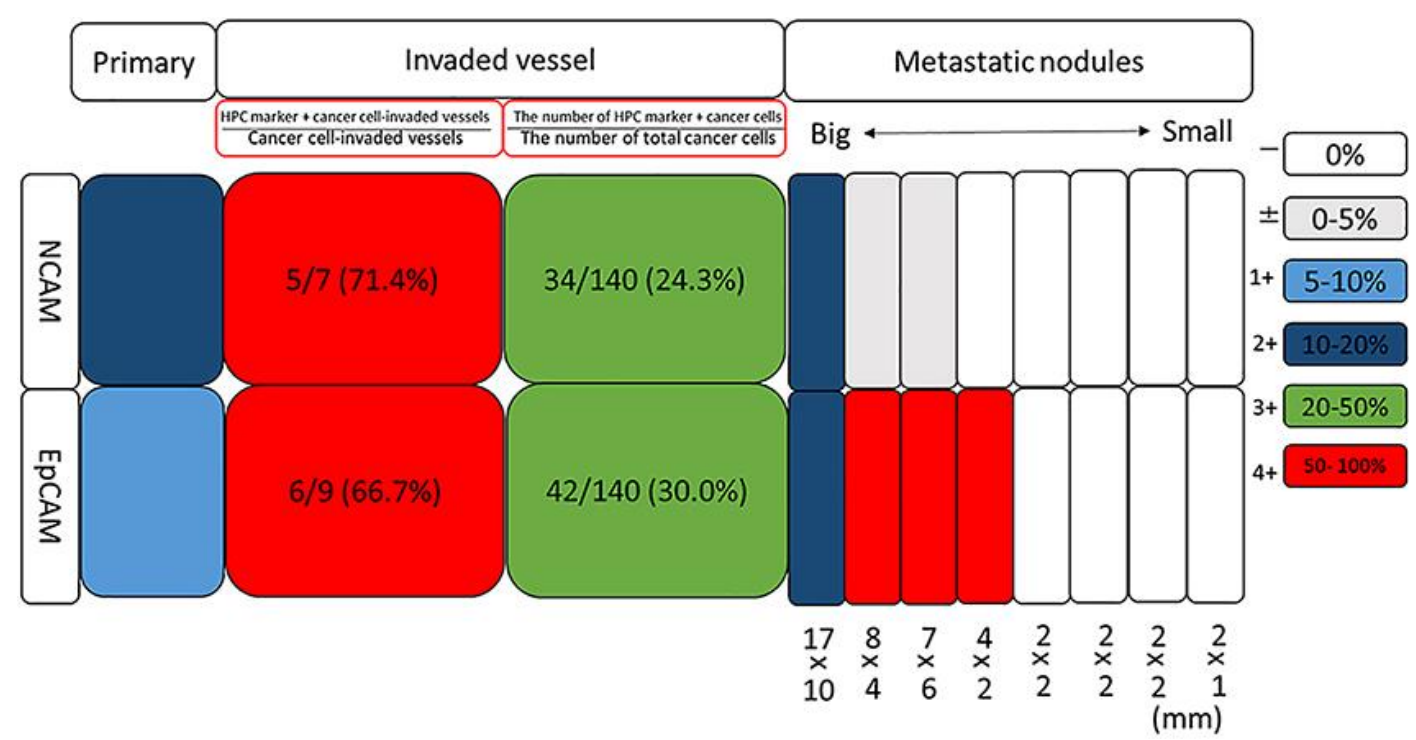

Fig. 2. Quantification of HPC marker-positive cancer cells in the primary tumor, tumor-invaded vessels, and metastatic nodules. In the primary lesion, the percentage of cancer cells that were EpCAM- or NCAMpositive was 5-10\% and 10-20\%, respectively. In the cancer-invaded blood vessels, both the ratio of the number of vessels invaded by specific HPC marker-positive cancer cells/the total number of vessels invaded by cancer cells and the ratio of the number of specific HPC marker-positive cancer cells in the vessel/the total number of cancer cells in the vessel were much higher than the corresponding ratios in the primary lesion. In the metastatic lesions, the frequency of EpCAM- or NCAM-positive cancer cells differed according to the tumor, and the bigger tumors tended to include HPC marker-positive cancer cells. 